\title{
Clusters Evolution at Early Stages of 1,3,5-Triamino-2,4,6-trinitrobenzene under Various Heating Conditions: A Molecular Reactive Force Field Study
}

Yushi Wen, XiangguiXue, Xinping Long, and Chaoyang Zhang*

Institute of Chemical Materials, China Academy of Engineering Physics (CAEP), P. O. Box 919-311, Mianyang, Sichuan 621900, China.

\section{Supporting Information}

\section{Table of Contents}

S1 The detailed information of the supercells.

S2 Bond order minimum values used to determine molecules and the products of heated TATB.

S3 The products of TATB heated under various conditions.

S4 Primary reactions under various heating conditions. 


\section{S1. The detailed information of the supercells}

Table s1. The detailed information of the supercells.

\begin{tabular}{|c|c|c|c|}
\hline supercells & \multicolumn{3}{|c|}{ TATB } \\
\hline enlargement times from unit cells & \multicolumn{3}{|c|}{$6 \times 6 \times 6$} \\
\hline \multirow{3}{*}{ Lattice Parameters } & $\mathrm{a}$ & $\mathrm{b}$ & $\mathrm{c}$ \\
\cline { 2 - 4 } & $54.06 \AA$ & $54.17 \AA$ & $40.87 \AA$ \\
\cline { 2 - 4 } & $\alpha$ & $\beta$ & $\gamma$ \\
\cline { 2 - 4 } & $108.58^{\circ}$ & $119.97^{\circ}$ & $91.82^{\circ}$ \\
\hline Amounts of molecules & \multicolumn{3}{|c}{432} \\
\hline Amounts of atoms & \multicolumn{3}{|c}{10368} \\
\hline
\end{tabular}

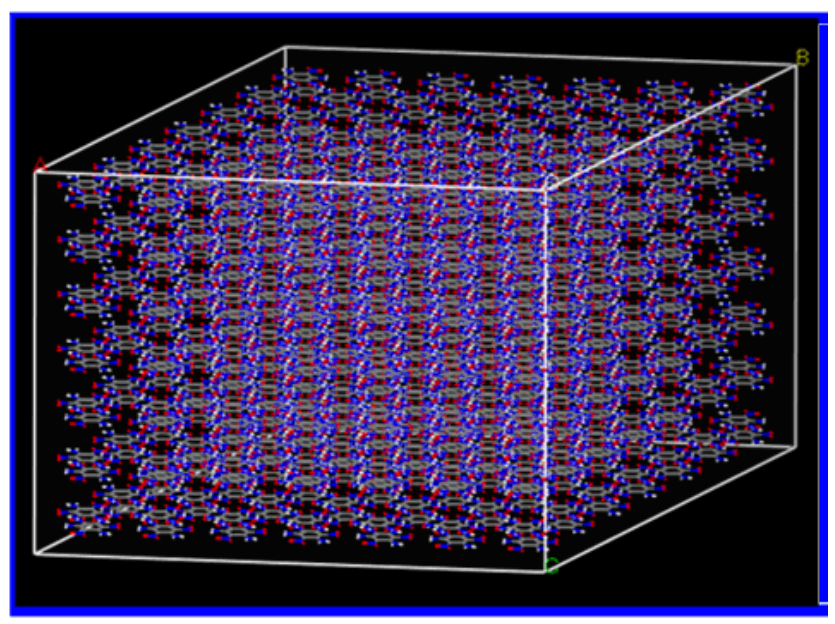

Fig. s1 Supercells of TATB. The carbon, hydrogen, oxygen and nitrogen atoms are indicated in grey, white, red and blue, respectively. 
S2. List of bond order minimum values used to determine molecules

Table s2. List of bond order minimum values used to determine molecules.

\begin{tabular}{|c|c|c|}
\hline Atom type & Atom type & Bond order \\
\hline $\mathrm{C}$ & $\mathrm{N}$ & 0.3 \\
\hline $\mathrm{C}$ & $\mathrm{C}$ & 0.55 \\
\hline $\mathrm{C}$ & $\mathrm{O}$ & 0.65 \\
\hline $\mathrm{C}$ & $\mathrm{H}$ & 0.4 \\
\hline $\mathrm{O}$ & $\mathrm{O}$ & 0.65 \\
\hline $\mathrm{N}$ & $\mathrm{O}$ & 0.4 \\
\hline $\mathrm{O}$ & $\mathrm{H}$ & 0.4 \\
\hline $\mathrm{H}$ & $\mathrm{H}$ & 0.55 \\
\hline $\mathrm{H}$ & $\mathrm{N}$ & 0.55 \\
\hline $\mathrm{N}$ & $\mathrm{N}$ & 0.55 \\
\hline
\end{tabular}


S3. The products of TATB heated under various conditions.

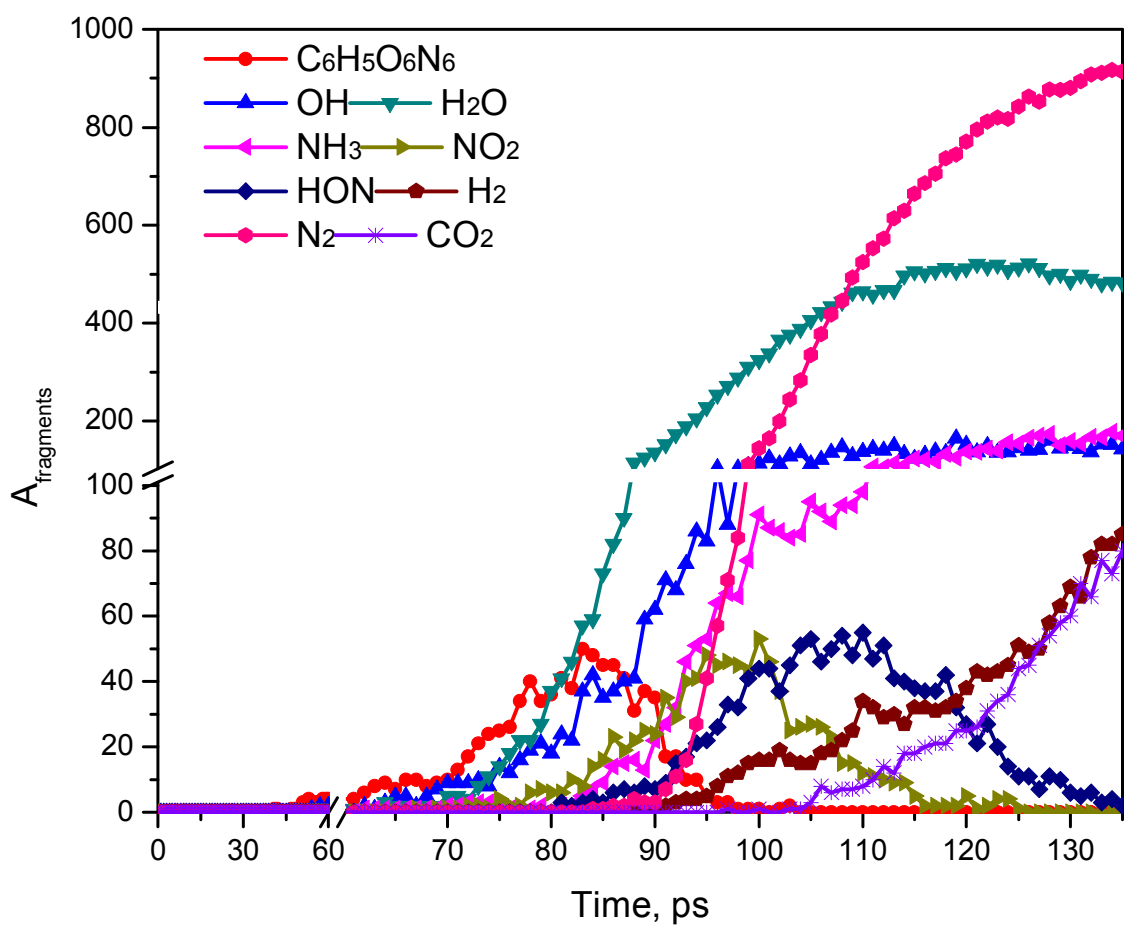

Fig. s2 The products of TATB programmed heated at a rate of $20 \mathrm{~K} / \mathrm{ps}$.

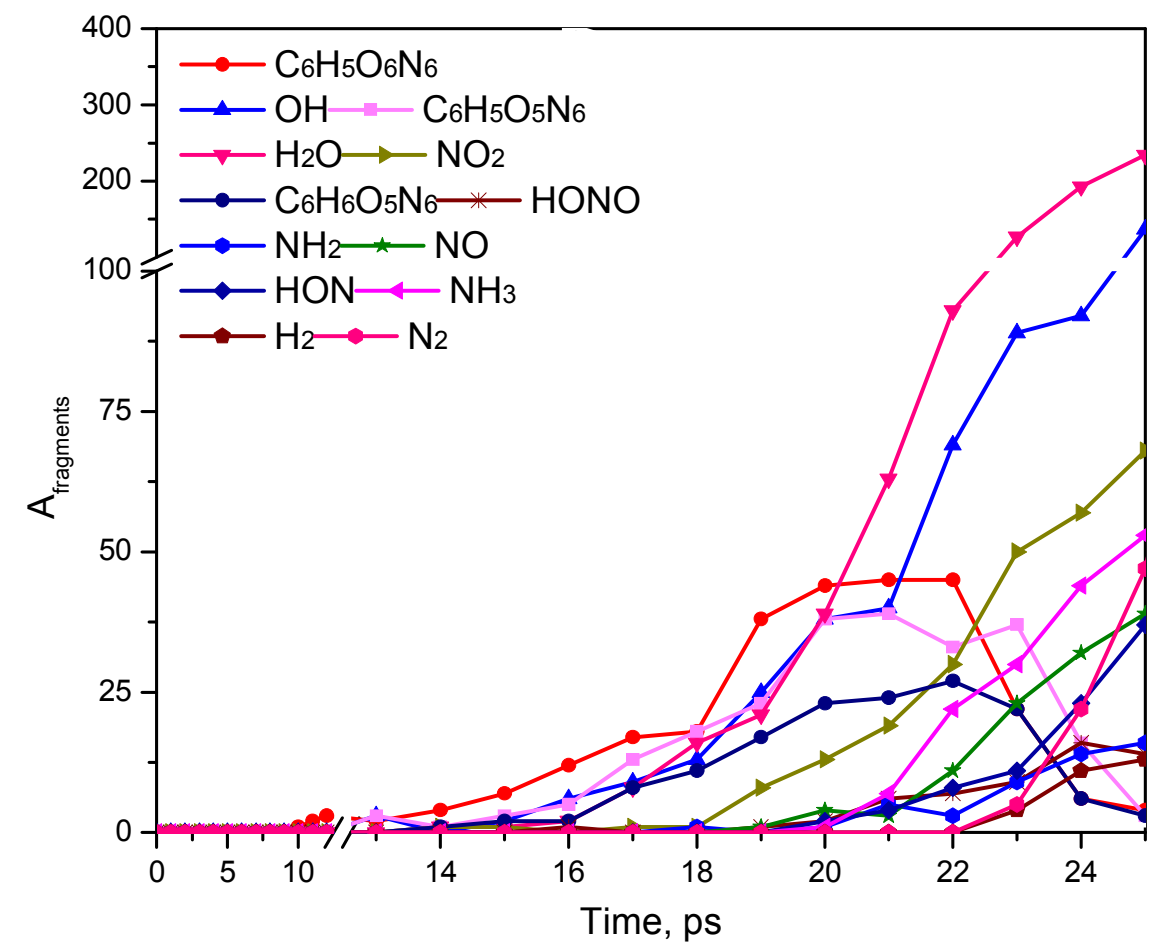

Fig. $\mathrm{s} 3$ The products of TATB programmed heated at a rate of $100 \mathrm{~K} / \mathrm{ps}$. 


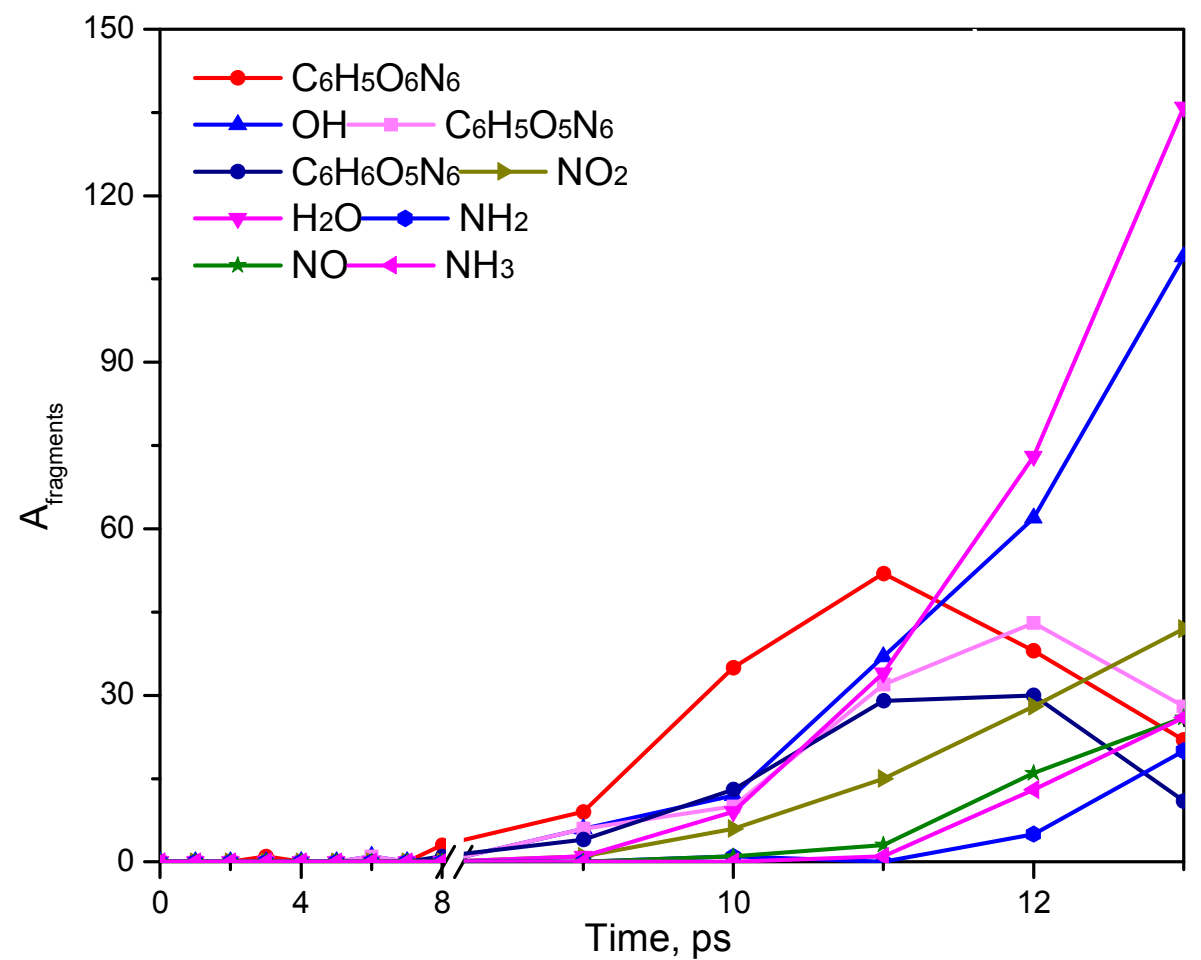

Fig. s4 The products of TATB programmed heated at a rate of $200 \mathrm{~K} / \mathrm{ps}$.

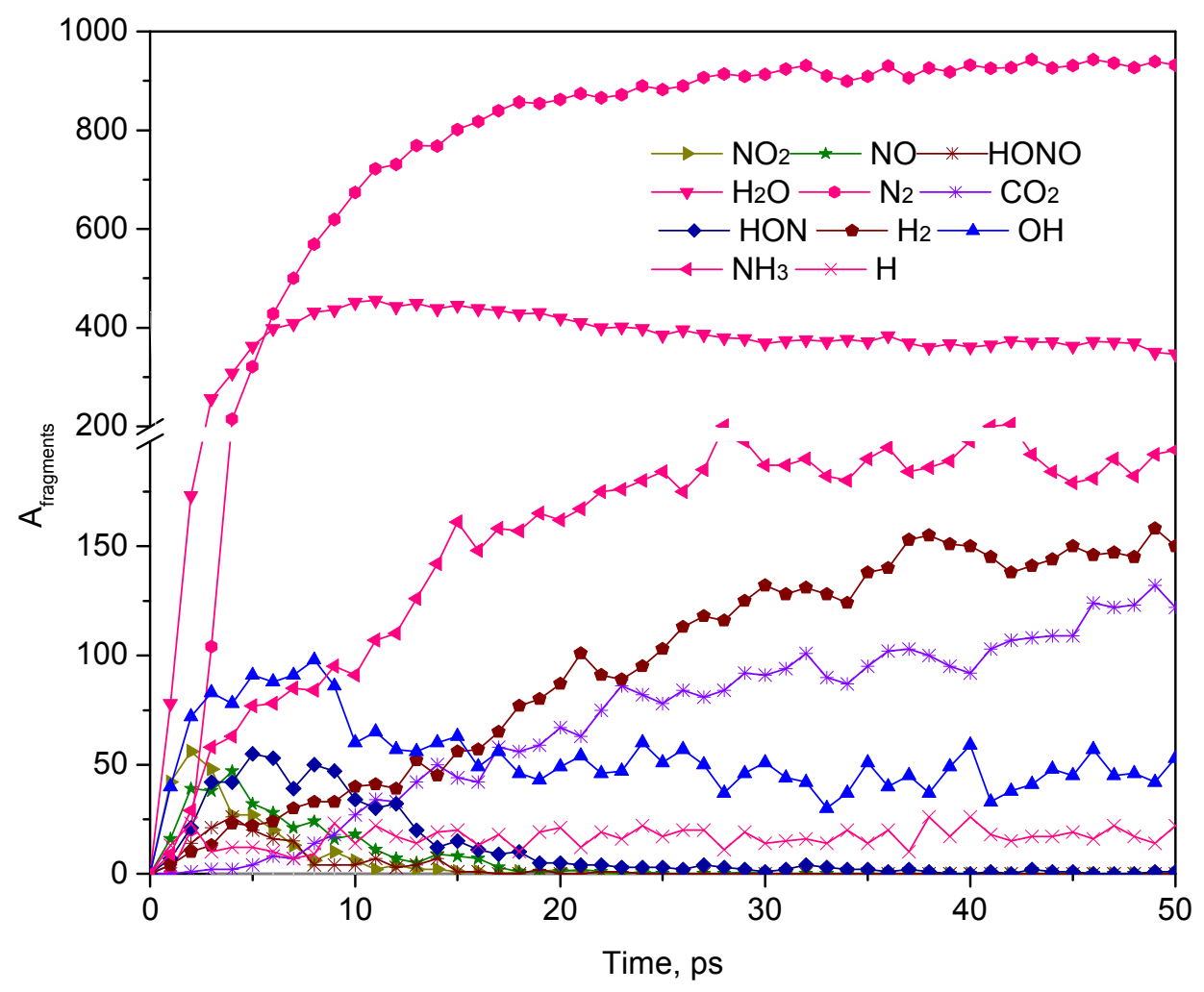

Fig. s5 The products of TATB heated at $3000 \mathrm{~K}$. 


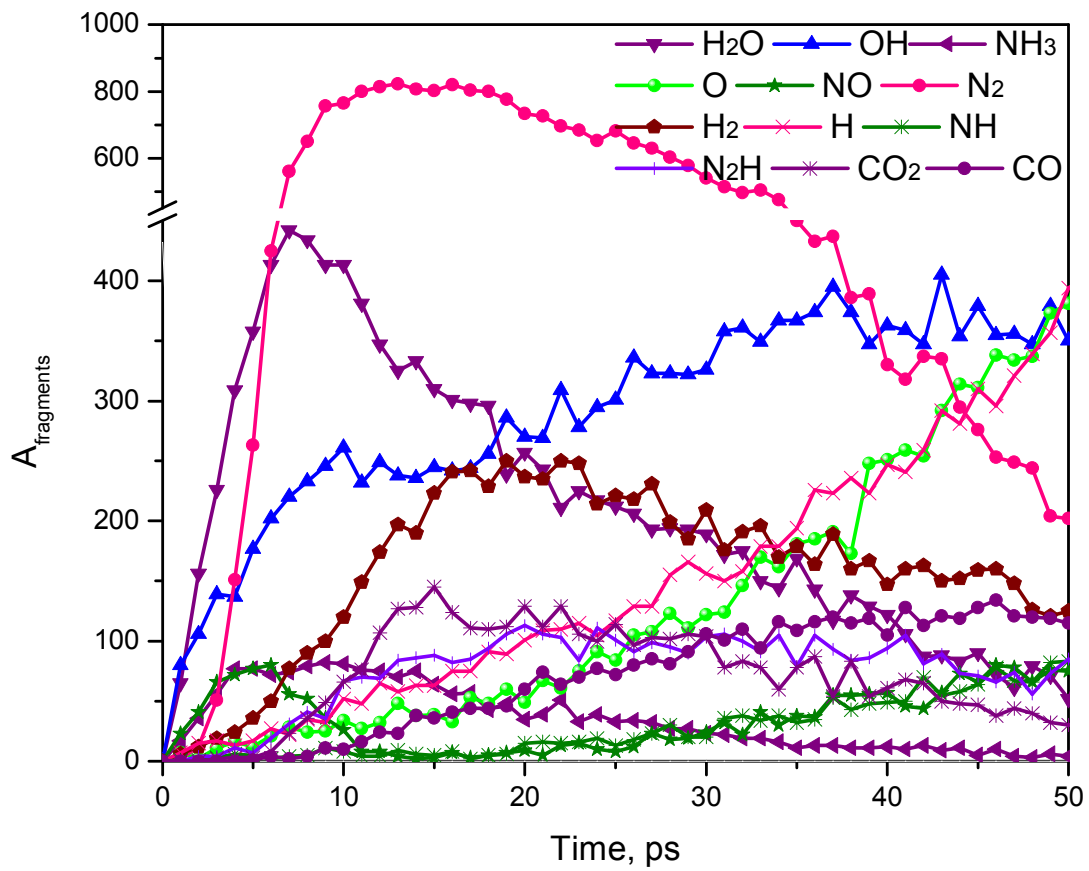

Fig. s6 The products of TATB adiabatic heated from $3000 \mathrm{~K}$. 
S4 Primary reactions under various heating conditions.

Table s3. Primary reactions and their frequencies under programmed heating with rate of $20 \mathrm{~K} / \mathrm{ps}$ (time of statistics is $0 \sim 135 \mathrm{ps}$ with an interval of $1 \mathrm{ps}$ ).

\begin{tabular}{|c|c|c|c|}
\hline Frequency & Reactants & & Products \\
\hline 31 & $\mathrm{C}_{6} \mathrm{H}_{6} \mathrm{O}_{6} \mathrm{~N}_{6}+\mathrm{C}_{6} \mathrm{H}_{6} \mathrm{O}_{6} \mathrm{~N}_{6}$ & $=>$ & $\mathrm{C}_{6} \mathrm{H}_{5} \mathrm{O}_{6} \mathrm{~N}_{6}+\mathrm{C}_{6} \mathrm{H}_{7} \mathrm{O}_{6} \mathrm{~N}_{6}$ \\
\hline 28 & $\mathrm{C}_{6} \mathrm{H}_{6} \mathrm{O}_{6} \mathrm{~N}_{6}$ & $\Rightarrow$ & $\mathrm{C}_{6} \mathrm{H}_{5} \mathrm{O}_{5} \mathrm{~N}_{6}+\mathrm{HO}$ \\
\hline 14 & $\mathrm{C}_{6} \mathrm{H}_{7} \mathrm{O}_{6} \mathrm{~N}_{6}$ & $=>$ & $\mathrm{C}_{6} \mathrm{H}_{6} \mathrm{O}_{5} \mathrm{~N}_{6}+\mathrm{HO}$ \\
\hline 10 & $\mathrm{C}_{6} \mathrm{H}_{6} \mathrm{O}_{6} \mathrm{~N}_{6}+\mathrm{HO}$ & $\Rightarrow$ & $\mathrm{C}_{6} \mathrm{H}_{5} \mathrm{O}_{6} \mathrm{~N}_{6}+\mathrm{H}_{2} \mathrm{O}$ \\
\hline 10 & $\mathrm{C}_{6} \mathrm{H}_{6} \mathrm{O}_{6} \mathrm{~N}_{6}+\mathrm{C}_{6} \mathrm{H}_{6} \mathrm{O}_{6} \mathrm{~N}_{6}$ & $\Rightarrow$ & $\mathrm{C}_{6} \mathrm{H}_{5} \mathrm{O}_{5} \mathrm{~N}_{6}+\mathrm{C}_{6} \mathrm{H}_{5} \mathrm{O}_{6} \mathrm{~N}_{6}+\mathrm{H}_{2} \mathrm{O}$ \\
\hline 9 & $\mathrm{C}_{6} \mathrm{H}_{6} \mathrm{O}_{6} \mathrm{~N}_{6}$ & $\Rightarrow$ & $\mathrm{C}_{6} \mathrm{H}_{4} \mathrm{O}_{5} \mathrm{~N}_{6}+\mathrm{H}_{2} \mathrm{O}$ \\
\hline 9 & $\mathrm{C}_{6} \mathrm{H}_{6} \mathrm{O}_{6} \mathrm{~N}_{6}+\mathrm{HO}$ & $=>$ & $\mathrm{C}_{6} \mathrm{H}_{7} \mathrm{O}_{7} \mathrm{~N}_{6}$ \\
\hline 9 & $\mathrm{C}_{6} \mathrm{H}_{7} \mathrm{O}_{6} \mathrm{~N}_{6}$ & $\Rightarrow$ & $\mathrm{C}_{6} \mathrm{H}_{5} \mathrm{O}_{5} \mathrm{~N}_{6}+\mathrm{H}_{2} \mathrm{O}$ \\
\hline 9 & $\mathrm{H}_{2} \mathrm{~N}_{2}$ & $=>$ & $\mathrm{H}_{2}+\mathrm{N}_{2}$ \\
\hline 8 & $\mathrm{H}_{2} \mathrm{~N}+\mathrm{H}_{2} \mathrm{O}$ & $\Rightarrow$ & $\mathrm{H}_{3} \mathrm{~N}+\mathrm{HO}$ \\
\hline 8 & $\mathrm{C}_{6} \mathrm{H}_{6} \mathrm{O}_{6} \mathrm{~N}_{6}+\mathrm{C}_{6} \mathrm{H}_{6} \mathrm{O}_{6} \mathrm{~N}_{6}$ & $\Rightarrow$ & $\mathrm{C}_{6} \mathrm{H}_{6} \mathrm{O}_{5} \mathrm{~N}_{6}+\mathrm{C}_{6} \mathrm{H}_{6} \mathrm{O}_{7} \mathrm{~N}_{6}$ \\
\hline 8 & $\mathrm{HO}+\mathrm{HO}$ & $\Rightarrow$ & $\mathrm{H}_{2} \mathrm{O}_{2}$ \\
\hline 6 & $\mathrm{H}+\mathrm{HO}$ & $=>$ & $\mathrm{H}_{2} \mathrm{O}$ \\
\hline 6 & $\mathrm{C}_{6} \mathrm{H}_{6} \mathrm{O}_{6} \mathrm{~N}_{6}+\mathrm{C}_{6} \mathrm{H}_{6} \mathrm{O}_{6} \mathrm{~N}_{6}$ & $=>$ & $\mathrm{C}_{6} \mathrm{H}_{5} \mathrm{O}_{6} \mathrm{~N}_{6}+\mathrm{C}_{6} \mathrm{H}_{6} \mathrm{O}_{5} \mathrm{~N}_{6}+\mathrm{HO}$ \\
\hline 5 & $\mathrm{HO}_{3} \mathrm{~N}$ & $\Rightarrow$ & $\mathrm{HO}+\mathrm{NO}_{2}$ \\
\hline
\end{tabular}


Table s4. Primary reactions and their frequencies under programmed heating with rate of $100 \mathrm{~K} / \mathrm{ps}$ (time of statistics is $0 \sim 27 \mathrm{ps}$ with an interval of $1 \mathrm{ps}$ )

\begin{tabular}{|c|c|c|}
\hline Frequency & Reactants & Products \\
\hline 28 & $\mathrm{C}_{6} \mathrm{H}_{6} \mathrm{O}_{6} \mathrm{~N}_{6}$ & $=>\quad \mathrm{C}_{6} \mathrm{H}_{5} \mathrm{O}_{5} \mathrm{~N}_{6}+\mathrm{HO}$ \\
\hline 17 & $\mathrm{C}_{6} \mathrm{H}_{6} \mathrm{O}_{6} \mathrm{~N}_{6}+\mathrm{C}_{6} \mathrm{H}_{6} \mathrm{O}_{6} \mathrm{~N}_{6}$ & $\mathrm{C}_{6} \mathrm{H}_{5} \mathrm{O}_{6} \mathrm{~N}_{6}+\mathrm{C}_{6} \mathrm{H}_{7} \mathrm{O}_{6} \mathrm{~N}_{6}$ \\
\hline 7 & $\mathrm{C}_{6} \mathrm{H}_{6} \mathrm{O}_{6} \mathrm{~N}_{6}+\mathrm{C}_{6} \mathrm{H}_{6} \mathrm{O}_{6} \mathrm{~N}_{6}$ & $\Rightarrow \quad \mathrm{C}_{6} \mathrm{H}_{5} \mathrm{O}_{5} \mathrm{~N}_{6}+\mathrm{C}_{6} \mathrm{H}_{5} \mathrm{O}_{6} \mathrm{~N}_{6}+\mathrm{H}_{2} \mathrm{O}$ \\
\hline 7 & $\mathrm{C}_{6} \mathrm{H}_{6} \mathrm{O}_{6} \mathrm{~N}_{6}+\mathrm{C}_{6} \mathrm{H}_{6} \mathrm{O}_{6} \mathrm{~N}_{6}$ & $\mathrm{C}_{6} \mathrm{H}_{6} \mathrm{O}_{5} \mathrm{~N}_{6}+\mathrm{C}_{6} \mathrm{H}_{6} \mathrm{O}_{7} \mathrm{~N}_{6}$ \\
\hline 6 & $\mathrm{C}_{6} \mathrm{H}_{6} \mathrm{O}_{6} \mathrm{~N}_{6}+\mathrm{HO}$ & $\Rightarrow \mathrm{C}_{6} \mathrm{H}_{5} \mathrm{O}_{6} \mathrm{~N}_{6}+\mathrm{H}_{2} \mathrm{O}$ \\
\hline 5 & $\mathrm{C}_{6} \mathrm{H}_{6} \mathrm{O}_{6} \mathrm{~N}_{6}+\mathrm{C}_{6} \mathrm{H}_{6} \mathrm{O}_{6} \mathrm{~N}_{6}$ & $\Rightarrow \quad \mathrm{C}_{6} \mathrm{H}_{5} \mathrm{O}_{5} \mathrm{~N}_{6}+\mathrm{C}_{6} \mathrm{H}_{7} \mathrm{O}_{7} \mathrm{~N}_{6}$ \\
\hline 5 & $\mathrm{C}_{6} \mathrm{H}_{6} \mathrm{O}_{6} \mathrm{~N}_{6}$ & $\mathrm{C}_{6} \mathrm{H}_{5} \mathrm{O}_{6} \mathrm{~N}_{6}+\mathrm{H}$ \\
\hline 5 & $\mathrm{C}_{6} \mathrm{H}_{7} \mathrm{O}_{7} \mathrm{~N}_{6}$ & $=>\quad \mathrm{C}_{6} \mathrm{H}_{6} \mathrm{O}_{6} \mathrm{~N}_{6}+\mathrm{HO}$ \\
\hline 5 & $\mathrm{C}_{6} \mathrm{H}_{7} \mathrm{O}_{6} \mathrm{~N}_{6}$ & $=>\quad \mathrm{C}_{6} \mathrm{H}_{6} \mathrm{O}_{5} \mathrm{~N}_{6}+\mathrm{HO}$ \\
\hline 4 & $\mathrm{C}_{6} \mathrm{H}_{6} \mathrm{O}_{6} \mathrm{~N}_{6}$ & $\Rightarrow \mathrm{C}_{6} \mathrm{H}_{6} \mathrm{O}_{4} \mathrm{~N}_{5}+\mathrm{NO}_{2}$ \\
\hline 4 & $\mathrm{C}_{6} \mathrm{H}_{6} \mathrm{O}_{6} \mathrm{~N}_{6}$ & $\Rightarrow \mathrm{C}_{6} \mathrm{H}_{4} \mathrm{O}_{5} \mathrm{~N}_{6}+\mathrm{H}_{2} \mathrm{O}$ \\
\hline 4 & $\mathrm{C}_{6} \mathrm{H}_{6} \mathrm{O}_{6} \mathrm{~N}_{6}+\mathrm{C}_{6} \mathrm{H}_{6} \mathrm{O}_{6} \mathrm{~N}_{6}$ & $\Rightarrow \quad \mathrm{C}_{12} \mathrm{H}_{12} \mathrm{O}_{12} \mathrm{~N}_{12}$ \\
\hline 4 & $\mathrm{C}_{6} \mathrm{H}_{6} \mathrm{O}_{7} \mathrm{~N}_{6}$ & $\Rightarrow \mathrm{C}_{6} \mathrm{H}_{6} \mathrm{O}_{5} \mathrm{~N}_{5}+\mathrm{NO}_{2}$ \\
\hline 4 & $\mathrm{C}_{6} \mathrm{H}_{5} \mathrm{O}_{5} \mathrm{~N}_{6}+\mathrm{C}_{6} \mathrm{H}_{6} \mathrm{O}_{6} \mathrm{~N}_{6}$ & $\Rightarrow \mathrm{C}_{6} \mathrm{H}_{5} \mathrm{O}_{6} \mathrm{~N}_{6}+\mathrm{C}_{6} \mathrm{H}_{6} \mathrm{O}_{5} \mathrm{~N}_{6}$ \\
\hline 4 & $\mathrm{C}_{6} \mathrm{H}_{6} \mathrm{O}_{6} \mathrm{~N}_{6}+\mathrm{C}_{6} \mathrm{H}_{6} \mathrm{O}_{6} \mathrm{~N}_{6}$ & $\Rightarrow \mathrm{C}_{6} \mathrm{H}_{5} \mathrm{O}_{6} \mathrm{~N}_{6}+\mathrm{C}_{6} \mathrm{H}_{6} \mathrm{O}_{5} \mathrm{~N}_{6}+\mathrm{HO}$ \\
\hline
\end{tabular}


Table s5. Primary reactions and their frequencies under programmed heating with rate of $200 \mathrm{~K} / \mathrm{ps}$ (time of statistics is $0 \sim 13.5 \mathrm{ps}$ with an interval of $1 \mathrm{ps}$ )

\begin{tabular}{|c|c|c|c|}
\hline Frequency & Reactants & & Products \\
\hline 29 & $\mathrm{C}_{6} \mathrm{H}_{6} \mathrm{O}_{6} \mathrm{~N}_{6}$ & $\Rightarrow$ & $\mathrm{C}_{6} \mathrm{H}_{5} \mathrm{O}_{5} \mathrm{~N}_{6}+\mathrm{HO}$ \\
\hline 21 & $\mathrm{C}_{6} \mathrm{H}_{6} \mathrm{O}_{6} \mathrm{~N}_{6}+\mathrm{C}_{6} \mathrm{H}_{6} \mathrm{O}_{6} \mathrm{~N}_{6}$ & $=>$ & $\mathrm{C}_{6} \mathrm{H}_{5} \mathrm{O}_{6} \mathrm{~N}_{6}+\mathrm{C}_{6} \mathrm{H}_{7} \mathrm{O}_{6} \mathrm{~N}_{6}$ \\
\hline 9 & $\mathrm{C}_{6} \mathrm{H}_{6} \mathrm{O}_{6} \mathrm{~N}_{6}$ & $\Rightarrow$ & $\mathrm{C}_{6} \mathrm{H}_{6} \mathrm{O}_{4} \mathrm{~N}_{5}+\mathrm{NO}_{2}$ \\
\hline 8 & $\mathrm{C}_{6} \mathrm{H}_{6} \mathrm{O}_{6} \mathrm{~N}_{6}+\mathrm{C}_{6} \mathrm{H}_{6} \mathrm{O}_{6} \mathrm{~N}_{6}$ & $\Rightarrow$ & $\mathrm{C}_{6} \mathrm{H}_{5} \mathrm{O}_{5} \mathrm{~N}_{6}+\mathrm{C}_{6} \mathrm{H}_{5} \mathrm{O}_{6} \mathrm{~N}_{6}+\mathrm{H}_{2} \mathrm{O}$ \\
\hline 8 & $\mathrm{C}_{6} \mathrm{H}_{6} \mathrm{O}_{6} \mathrm{~N}_{6}$ & $\Rightarrow$ & $\mathrm{C}_{6} \mathrm{H}_{5} \mathrm{O}_{6} \mathrm{~N}_{6}+\mathrm{H}$ \\
\hline 8 & $\mathrm{C}_{6} \mathrm{H}_{6} \mathrm{O}_{6} \mathrm{~N}_{6}+\mathrm{C}_{6} \mathrm{H}_{6} \mathrm{O}_{6} \mathrm{~N}_{6}$ & $\Rightarrow$ & $\mathrm{C}_{6} \mathrm{H}_{5} \mathrm{O}_{6} \mathrm{~N}_{6}+\mathrm{C}_{6} \mathrm{H}_{6} \mathrm{O}_{5} \mathrm{~N}_{6}+\mathrm{HO}$ \\
\hline 7 & $\mathrm{C}_{6} \mathrm{H}_{6} \mathrm{O}_{6} \mathrm{~N}_{6}+\mathrm{C}_{6} \mathrm{H}_{6} \mathrm{O}_{6} \mathrm{~N}_{6}$ & $\Rightarrow$ & $\mathrm{C}_{6} \mathrm{H}_{6} \mathrm{O}_{5} \mathrm{~N}_{6}+\mathrm{C}_{6} \mathrm{H}_{6} \mathrm{O}_{7} \mathrm{~N}_{6}$ \\
\hline 5 & $\mathrm{C}_{6} \mathrm{H}_{6} \mathrm{O}_{6} \mathrm{~N}_{6}+\mathrm{C}_{6} \mathrm{H}_{6} \mathrm{O}_{6} \mathrm{~N}_{6}$ & $\Rightarrow$ & $\mathrm{C}_{12} \mathrm{H}_{12} \mathrm{O}_{12} \mathrm{~N}_{12}$ \\
\hline 3 & $\mathrm{C}_{6} \mathrm{H}_{7} \mathrm{O}_{6} \mathrm{~N}_{6}$ & $\Rightarrow$ & $\mathrm{C}_{6} \mathrm{H}_{5} \mathrm{O}_{5} \mathrm{~N}_{6}+\mathrm{H}_{2} \mathrm{O}$ \\
\hline 3 & $\mathrm{C}_{6} \mathrm{H}_{6} \mathrm{O}_{6} \mathrm{~N}_{6}+\mathrm{C}_{6} \mathrm{H}_{7} \mathrm{O}_{6} \mathrm{~N}_{6}$ & $\Rightarrow$ & $\mathrm{C}_{6} \mathrm{H}_{5} \mathrm{O}_{6} \mathrm{~N}_{6}+\mathrm{C}_{6} \mathrm{H}_{6} \mathrm{O}_{5} \mathrm{~N}_{6}+\mathrm{H}_{2} \mathrm{O}$ \\
\hline 3 & $3 \mathrm{C}_{6} \mathrm{H}_{6} \mathrm{O}_{6} \mathrm{~N}_{6}$ & $\Rightarrow$ & $\mathrm{C}_{6} \mathrm{H}_{5} \mathrm{O}_{6} \mathrm{~N}_{6}+\mathrm{C}_{6} \mathrm{H}_{6} \mathrm{O}_{5} \mathrm{~N}_{6}+\mathrm{C}_{6} \mathrm{H}_{7} \mathrm{O}_{7} \mathrm{~N}_{6}$ \\
\hline 2 & $\mathrm{C}_{6} \mathrm{H}_{6} \mathrm{O}_{6} \mathrm{~N}_{6}+\mathrm{HO}$ & $\Rightarrow$ & $\mathrm{C}_{6} \mathrm{H}_{4} \mathrm{O}_{7} \mathrm{~N}_{5}+\mathrm{NH}_{3}$ \\
\hline 2 & $\mathrm{C}_{6} \mathrm{H}_{5} \mathrm{O}_{5} \mathrm{~N}_{6}+\mathrm{C}_{6} \mathrm{H}_{6} \mathrm{O}_{6} \mathrm{~N}_{6}$ & $\Rightarrow$ & $\mathrm{C}_{6} \mathrm{H}_{5} \mathrm{O}_{4} \mathrm{~N}_{6}+\mathrm{C}_{6} \mathrm{H}_{5} \mathrm{O}_{6} \mathrm{~N}_{6}+\mathrm{HO}$ \\
\hline 2 & $\mathrm{C}_{6} \mathrm{H}_{6} \mathrm{O}_{6} \mathrm{~N}_{6}+\mathrm{C}_{6} \mathrm{H}_{6} \mathrm{O}_{6} \mathrm{~N}_{6}$ & $\Rightarrow$ & $\mathrm{C}_{6} \mathrm{H}_{6} \mathrm{O}_{5} \mathrm{~N}_{5}+\mathrm{C}_{6} \mathrm{H}_{6} \mathrm{O}_{5} \mathrm{~N}_{6}+\mathrm{NO}_{2}$ \\
\hline 2 & $\mathrm{C}_{6} \mathrm{H}_{5} \mathrm{O}_{6} \mathrm{~N}_{6}+\mathrm{H}$ & $\Rightarrow$ & $\mathrm{C}_{6} \mathrm{H}_{5} \mathrm{O}_{5} \mathrm{~N}_{6}+\mathrm{HO}$ \\
\hline
\end{tabular}


Table s6. Primary reactions and their frequencies under constant temperature heating ( $\mathrm{T}=3000 \mathrm{~K}$ and the time of statistics is $0 \sim 5 \mathrm{ps}$ with an interval of $0.1 \mathrm{ps}$ )

\begin{tabular}{|c|c|c|c|}
\hline Frequency & Reactants & & Products \\
\hline 22 & $\mathrm{C}_{6} \mathrm{H}_{6} \mathrm{O}_{6} \mathrm{~N}_{6}$ & $=>$ & $\mathrm{C}_{6} \mathrm{H}_{6} \mathrm{O}_{4} \mathrm{~N}_{5}+\mathrm{NO}_{2}$ \\
\hline 21 & $\mathrm{C}_{6} \mathrm{H}_{6} \mathrm{O}_{6} \mathrm{~N}_{6}+\mathrm{C}_{6} \mathrm{H}_{6} \mathrm{O}_{6} \mathrm{~N}_{6}$ & $\Rightarrow$ & $\mathrm{C}_{6} \mathrm{H}_{5} \mathrm{O}_{6} \mathrm{~N}_{6}+\mathrm{C}_{6} \mathrm{H}_{7} \mathrm{O}_{6} \mathrm{~N}_{6}$ \\
\hline 18 & $\mathrm{C}_{6} \mathrm{H}_{6} \mathrm{O}_{6} \mathrm{~N}_{6}$ & $\Rightarrow$ & $\mathrm{C}_{6} \mathrm{H}_{5} \mathrm{O}_{5} \mathrm{~N}_{6}+\mathrm{HO}$ \\
\hline 15 & $\mathrm{C}_{6} \mathrm{H}_{6} \mathrm{O}_{6} \mathrm{~N}_{6}$ & $\Rightarrow$ & $\mathrm{C}_{6} \mathrm{H}_{5} \mathrm{O}_{6} \mathrm{~N}_{6}+\mathrm{H}$ \\
\hline 8 & $\mathrm{C}_{6} \mathrm{H}_{6} \mathrm{O}_{6} \mathrm{~N}_{6}$ & $\Rightarrow$ & $\mathrm{C}_{6} \mathrm{H}_{6} \mathrm{O}_{5} \mathrm{~N}_{6}+\mathrm{O}$ \\
\hline 6 & $\mathrm{C}_{6} \mathrm{H}_{6} \mathrm{O}_{6} \mathrm{~N}_{6}$ & $\Rightarrow$ & $\mathrm{C}_{6} \mathrm{H}_{4} \mathrm{O}_{6} \mathrm{~N}_{5}+\mathrm{H}_{2} \mathrm{~N}$ \\
\hline 6 & $\mathrm{C}_{6} \mathrm{H}_{6} \mathrm{O}_{6} \mathrm{~N}_{6}+\mathrm{C}_{6} \mathrm{H}_{6} \mathrm{O}_{6} \mathrm{~N}_{6}$ & $\Rightarrow$ & $\mathrm{C}_{6} \mathrm{H}_{5} \mathrm{O}_{5} \mathrm{~N}_{6}+\mathrm{C}_{6} \mathrm{H}_{5} \mathrm{O}_{6} \mathrm{~N}_{6}+\mathrm{H}_{2} \mathrm{O}$ \\
\hline 5 & $\mathrm{C}_{6} \mathrm{H}_{6} \mathrm{O}_{6} \mathrm{~N}_{6}+\mathrm{C}_{6} \mathrm{H}_{6} \mathrm{O}_{6} \mathrm{~N}_{6}$ & $\Rightarrow$ & $\mathrm{C}_{12} \mathrm{H}_{12} \mathrm{O}_{12} \mathrm{~N}_{12}$ \\
\hline 5 & $\mathrm{C}_{6} \mathrm{H}_{6} \mathrm{O}_{6} \mathrm{~N}_{6}+\mathrm{C}_{6} \mathrm{H}_{6} \mathrm{O}_{6} \mathrm{~N}_{6}$ & $\Rightarrow$ & $\mathrm{C}_{6} \mathrm{H}_{6} \mathrm{O}_{5} \mathrm{~N}_{6}+\mathrm{C}_{6} \mathrm{H}_{6} \mathrm{O}_{7} \mathrm{~N}_{6}$ \\
\hline 5 & $\mathrm{C}_{6} \mathrm{H}_{6} \mathrm{O}_{6} \mathrm{~N}_{6}+\mathrm{C}_{6} \mathrm{H}_{6} \mathrm{O}_{6} \mathrm{~N}_{6}$ & $\Rightarrow$ & $\mathrm{C}_{6} \mathrm{H}_{5} \mathrm{O}_{6} \mathrm{~N}_{6}+\mathrm{C}_{6} \mathrm{H}_{6} \mathrm{O}_{5} \mathrm{~N}_{6}+\mathrm{HO}$ \\
\hline 5 & $\mathrm{C}_{6} \mathrm{H}_{6} \mathrm{O}_{6} \mathrm{~N}_{6}+\mathrm{O}$ & $\Rightarrow$ & $\mathrm{C}_{6} \mathrm{H}_{6} \mathrm{O}_{7} \mathrm{~N}_{6}$ \\
\hline 5 & $\mathrm{C}_{6} \mathrm{H}_{6} \mathrm{O}_{6} \mathrm{~N}_{6}+\mathrm{HO}$ & $\Rightarrow$ & $\mathrm{C}_{6} \mathrm{H}_{5} \mathrm{O}_{6} \mathrm{~N}_{6}+\mathrm{H}_{2} \mathrm{O}$ \\
\hline 4 & $\mathrm{C}_{6} \mathrm{H}_{7} \mathrm{O}_{6} \mathrm{~N}_{6}$ & $\Rightarrow$ & $\mathrm{C}_{6} \mathrm{H}_{4} \mathrm{O}_{6} \mathrm{~N}_{5}+\mathrm{NH}_{3}$ \\
\hline 4 & $\mathrm{C}_{6} \mathrm{H}_{5} \mathrm{O}_{6} \mathrm{~N}_{6}$ & $\Rightarrow$ & $\mathrm{C}_{6} \mathrm{H}_{4} \mathrm{O}_{5} \mathrm{~N}_{6}+\mathrm{HO}$ \\
\hline 4 & $\mathrm{C}_{6} \mathrm{H}_{6} \mathrm{O}_{6} \mathrm{~N}_{6}+\mathrm{H}$ & $\Rightarrow$ & $\mathrm{C}_{6} \mathrm{H}_{6} \mathrm{O}_{5} \mathrm{~N}_{6}+\mathrm{HO}$ \\
\hline
\end{tabular}


Table s7. Primary reactions and their frequencies under adiabatic heating with preheating at $3000 \mathrm{~K}$ for 0.5 ps (the time of statistics is $0 \sim 5 \mathrm{ps}$ with an interval of $0.1 \mathrm{ps}$ )

\begin{tabular}{|c|c|c|c|}
\hline Frequency & Reactants & & Products \\
\hline 33 & $\mathrm{C}_{6} \mathrm{H}_{6} \mathrm{O}_{6} \mathrm{~N}_{6}$ & $=>$ & $\mathrm{C}_{6} \mathrm{H}_{5} \mathrm{O}_{5} \mathrm{~N}_{6}+\mathrm{HO}$ \\
\hline 16 & $\mathrm{C}_{6} \mathrm{H}_{6} \mathrm{O}_{6} \mathrm{~N}_{6}$ & $=>$ & $\mathrm{C}_{6} \mathrm{H}_{6} \mathrm{O}_{4} \mathrm{~N}_{5}+\mathrm{NO}_{2}$ \\
\hline 13 & $\mathrm{C}_{6} \mathrm{H}_{6} \mathrm{O}_{6} \mathrm{~N}_{6}+\mathrm{HO}$ & $=>$ & $\mathrm{C}_{6} \mathrm{H}_{7} \mathrm{O}_{7} \mathrm{~N}_{6}$ \\
\hline 11 & $\mathrm{C}_{6} \mathrm{H}_{7} \mathrm{O}_{6} \mathrm{~N}_{6}$ & $=>$ & $\mathrm{C}_{6} \mathrm{H}_{6} \mathrm{O}_{5} \mathrm{~N}_{6}+\mathrm{HO}$ \\
\hline 11 & $\mathrm{C}_{6} \mathrm{H}_{6} \mathrm{O}_{6} \mathrm{~N}_{6}+\mathrm{C}_{6} \mathrm{H}_{6} \mathrm{O}_{6} \mathrm{~N}_{6}$ & $=>$ & $\mathrm{C}_{6} \mathrm{H}_{5} \mathrm{O}_{6} \mathrm{~N}_{6}+\mathrm{C}_{6} \mathrm{H}_{7} \mathrm{O}_{6} \mathrm{~N}_{6}$ \\
\hline 8 & $\mathrm{C}_{6} \mathrm{H}_{5} \mathrm{O}_{5} \mathrm{~N}_{6}$ & $=>$ & $\mathrm{C}_{6} \mathrm{H}_{4} \mathrm{O}_{4} \mathrm{~N}_{6}+\mathrm{HO}$ \\
\hline 6 & $\mathrm{C}_{6} \mathrm{H}_{5} \mathrm{O}_{6} \mathrm{~N}_{6}+\mathrm{HO}$ & $\Rightarrow>$ & $\mathrm{C}_{6} \mathrm{H}_{4} \mathrm{O}_{6} \mathrm{~N}_{6}+\mathrm{H}_{2} \mathrm{O}$ \\
\hline 6 & $\mathrm{C}_{6} \mathrm{H}_{5} \mathrm{O}_{6} \mathrm{~N}_{6}+\mathrm{C}_{6} \mathrm{H}_{6} \mathrm{O}_{6} \mathrm{~N}_{6}$ & $=>$ & $\mathrm{C}_{12} \mathrm{H}_{11} \mathrm{O}_{12} \mathrm{~N}_{12}$ \\
\hline 6 & $\mathrm{C}_{6} \mathrm{H}_{6} \mathrm{O}_{6} \mathrm{~N}_{6}+\mathrm{H}$ & $=>$ & $\mathrm{C}_{6} \mathrm{H}_{7} \mathrm{O}_{6} \mathrm{~N}_{6}$ \\
\hline 5 & $\mathrm{C}_{6} \mathrm{H}_{6} \mathrm{O}_{7} \mathrm{~N}_{6}$ & $=>$ & $\mathrm{C}_{6} \mathrm{H}_{6} \mathrm{O}_{5} \mathrm{~N}_{5}+\mathrm{NO}_{2}$ \\
\hline 5 & $\mathrm{C}_{6} \mathrm{H}_{7} \mathrm{O}_{6} \mathrm{~N}_{6}$ & $=>$ & $\mathrm{C}_{6} \mathrm{H}_{5} \mathrm{O}_{5} \mathrm{~N}_{6}+\mathrm{H}_{2} \mathrm{O}$ \\
\hline 5 & $\mathrm{C}_{6} \mathrm{H}_{6} \mathrm{O}_{5} \mathrm{~N}_{6}$ & $=>$ & $\mathrm{C}_{6} \mathrm{H}_{5} \mathrm{O}_{4} \mathrm{~N}_{6}+\mathrm{HO}$ \\
\hline 5 & $\mathrm{C}_{6} \mathrm{H}_{5} \mathrm{O}_{5} \mathrm{~N}_{6}$ & $=>$ & $\mathrm{C}_{6} \mathrm{H}_{5} \mathrm{O}_{3} \mathrm{~N}_{5}+\mathrm{NO}_{2}$ \\
\hline 5 & $\mathrm{C}_{6} \mathrm{H}_{4} \mathrm{O}_{4} \mathrm{~N}_{4}$ & $=>$ & $\mathrm{C}_{6} \mathrm{H}_{3} \mathrm{O}_{3} \mathrm{~N}_{4}+\mathrm{HO}$ \\
\hline 5 & $\mathrm{O}_{2} \mathrm{~N}$ & $=>$ & $\mathrm{O}+\mathrm{NO}$ \\
\hline
\end{tabular}

